\title{
To Treat or Not to Treat? Effect of Urate-Lowering Therapy on Renal Function, Blood Pressure and Safety in Patients with Asymptomatic Hyperuricemia: A Systematic Review and Network Meta-Analysis
}

\author{
Yu-Yu Tien, MD, MS, Ming-Chieb Shib, MD, PhD, Chiao-Pang Tien, MD, \\ Huei-Kai Huang, MD, and Yu-Kang Tu, DDS, PhD
}

Purpose: Hyperuricemia is associated with increased cardiovascular risk. Because patients with asymptomatic hyperuricemia ( $\mathrm{AH})$ experience no immediate discomfort and there are possible side effects of urate-lowering drugs, treatment for $\mathrm{AH}$ is controversial. We aimed to perform a network meta-analysis (NMA) to investigate the effects of different urate-lowering therapies (ULTs) on serum uric acid level, renal function, blood pressure (BP), and safety in AH patients.

Metbods: This NMA focused on AH patients. The intervention group (patients receiving urate-lowering drugs) was compared with others using other types of drugs, placebo, or usual care. We undertook a NMA under the frequentist framework by $R$.

Results: Thirteen eligible trials were identified. The interventions included allopurinol, febuxostat, and benzbromarone, which are not approved in the United States. Benzbromarone and allopurinol had the best efficacy on lowering serum uric acid level in short-term and long-term follow-up (mean difference $[\mathrm{MD}]=-3.05 ; 95 \% \mathrm{CI},-5.19$ to -0.91 vs $\mathrm{MD}=-3.17 ; 95 \% \mathrm{CI},-5.19$ to -1.15$)$. Patients using allopurinol had significantly higher eGFR than using placebo in both short-term and long-term follow-up (MD = 3.07; 95\% CI, 0.18 to 5.95 vs MD $=4.10 ; 95 \%$ CI, 2.66 to 5.54). No difference in BP was found between groups, except for febuxostat to diastolic BP after long-term treatment (MD $=-1.47 ; 95 \%$ CI, -2.91 to $-0.04)$. No statistically increased odds of safety events were found with the use of ULT.

Conclusions: Our result showed that in AH patients, allopurinol has a renoprotective effect. Febuxostat has a significant impact in lowering diastolic BP. ULT does not result in a higher risk of safety events. (J Am Board Fam Med 2022;35:140-151.)

Keywords: Asymptomatic Hyperuricemia, Blood Pressure, Disease Management, Family Medicine, Network Meta-Analysis, Serum Uric Acid, Systematic Review, Renal Function

\section{Introduction}

Vascular endothelium, a monolayer of endothelial cells, controls vascular tone and maintains vascular homeostasis, allowing it to maintain normal

This article was externally peer reviewed.

Submitted 3 July 2021; revised 8 September 2021 and 13 September 2021; accepted 15 September 2021.

From the Department of Family Medicine, Hsinchu Cathay General Hospital, Hsinchu, Taiwan (YYT); Institute of Epidemiology and Preventive Medicine, College of Public Health, National Taiwan University, Taipei, Taiwan (YYT, MCS, HKH, YKT); Department of Nephrology, Taipei City Hospital, Renai Branch, Taipei, Taiwan (CPT); Department of Family Medicine, Hualian Tzu Chi Hospital, Buddhist Tzu Chi Medical Foundation, Hualien, Taiwan (HKH); Department of Family Medicine, Cathay General Hospital, Taipei, Taiwan (YYT); Department of Dentistry, National Taiwan University Hospital, National Taiwan University, Taipei, Taiwan (YKT).

Funding: None.

Conflict of interest: None. physiologic mechanisms. ${ }^{1}$ Endothelial dysfunction means endothelial cells lose their normal function and is found to be associated with hypertension and chronic kidney disease (CKD) ${ }^{2,3}$ Hyperuricemia is 1 of its causes, and urate-lowering therapy (ULT) is proved to improve endothelial function. ${ }^{4-7}$ Therefore, many trials investigated whether patients under ULT attained better blood pressure (BP) control and renal function. ${ }^{8-10}$ ULT is commonly prescribed for patients if any symptom or sign of hyperuricemia develops.

Corresponding author: Professor Yu-Kang Tu, DDS, PhD, Institute of Epidemiology \& Preventive Medicine, College of Public Health, National Taiwan University, Taipei, Taiwan, 17 Xu-Zhou Road, Taipei, Taiwan (E-mail: yukangtu@ntu.edu.tw). 
However, more than half of hyperuricemic individuals remain asymptomatic. ${ }^{11}$ Asymptomatic hyperuricemia $(\mathrm{AH})$ is defined as hyperuricemic patients without either symptoms or signs of gout, tophi, hyperuricemic nephropathy, or uric acid nephrolithiasis. ${ }^{12}$ Because there are possible side effects of urate-lowering drugs, treatment for $\mathrm{AH}$ is controversial. ${ }^{13,14}$ Urate-lowering drugs include xanthine oxidase inhibitors, such as allopurinol and febuxostat, and uricosuric agents, such as benzbromarone and probenecid. Severe skin reaction, higher cardiovascular (CV) risk or impaired liver function related to those drugs have been reported. ${ }^{15-19}$ Benzbromarone was, therefore, withdrawn from the market in 2003 and has never been approved in the United States due to its reports of hepatotoxicity. ${ }^{20,21}$ Japanese guidelines for managing hyperuricemia and gout recommend initiating ULT for AH when serum urate levels increase to $>8.0 \mathrm{mg} / \mathrm{dL}$. ${ }^{22}$ However, this approach is not recommended in the United States and Europe owing to the side effects of these drugs. ${ }^{14}$

Xanthine oxidase inhibitors are thought to have the potency to decrease oxidative stress causing endothelial dysfunction. ${ }^{10,23}$ The metabolite of allopurinol is excreted predominantly by the kidney, and febuxostat is believed to be safe for patients with CKD owing to its hepatic elimination. ${ }^{24}$ The comparative effects of these drugs have not been investigated.

Network meta-analysis (NMA) is, therefore, a useful tool because it can use both direct and indirect evidence to compare the effects of all ULT. In contrast, previous meta-analyses either considered ULT as a single group or compared each drug to the control separately. Therefore, we conducted a systematic review and NMA to investigate the effects of different urate-lowering drugs on serum uric acid level, renal function, and BP in patients with $\mathrm{AH}$. We would also investigate the safety of those treatments to attain a balanced consideration for AH patients.

\section{Methods}

We conducted a systematic review and NMA of randomized controlled trials on patients with $\mathrm{AH}$. The intervention group (patients receiving uratelowering drugs) was compared with groups of other types of urate-lowering drugs, placebo, or usual care. The outcomes were serum uric acid level, renal function, $\mathrm{BP}$, and adverse events. We registered our systematic review on PROSPERO website. This NMA followed the preferred reporting items for systematic reviews and metaanalyses (PRISMA) extension guideline, which incorporated NMA for health care interventions and was registered in PROSPERO (registration number: CRD42021256528).

\section{Literature Search}

Two investigators (YYT and CPT) independently searched PubMed and Embase from their inception through October 8, 2020. We had also searched at ClinicalTrials. gov and hand-searched reference lists of relevant publications. The population of included trials was AH patients. Given that there are some controversies over the definition of hyperuricemia, we respected authors' definition of hyperuricemia in each study. ${ }^{12}$ If "asymptomatic" was not used to describe its population, a trial was still considered eligible if it enrolled patients without a history of gout or other related symptoms. Chronic hyperuricemic nephropathy is usually asymptomatic and is not easy to diagnose. If a trial described its patients as $\mathrm{AH}$ and with $C K D$, this was interpreted as that CKD in those patients was not caused by their hyperuricemia. Therefore, those studies would still be included. We used the keywords "hyperuricemia," "asymptomatic," "urate-lowering therapy," and classification or name of the drugs for searching. The search details are shown in Appendix 1. The bibliographies of recent review articles and previous meta-analyses were also manually searched for relevant studies.

\section{Study Outcome}

The primary outcomes were serum uric acid level, measured in units of $\mathrm{mg} / \mathrm{dL}$, renal function, assessed by estimated glomerular filtration rate (eGFR), and BP, measured in units of $\mathrm{mmH}, \mathrm{g}$ and divided to systolic and diastolic BP. The eGFR was calculated with 1 of the following methods: Cockcroft-Gault formula, the 4-variable modification of diet in renal disease study equation, or CKD epidemiology collaboration equation. The secondary outcome was adverse events, including the occurrence of impaired liver function, gastrointestinal event, CV event, skin reaction, and musculoskeletal event in patients within the trials identified by our search strategy. 


\section{Study Selection}

All titles and abstracts retrieved from the literature search were screened by 2 reviewers to determine the eligibility of a study. We included clinical trials where patients were randomly allocated to receive different treatments or placebo/usual care groups. We excluded conference proceedings without full text, nonrandomized controlled trials, the intervention group not receiving approved medicine, and studies not specific to asymptomatic adults.

\section{Data Extraction}

The outcomes were extracted independently from the included studies by 2 investigators mentioned above. For the primary outcomes, we evaluated the treatment effect by dividing the duration of treatment into short-term ( $\leq 6$ months) and long-term follow-up ( $>6$ months). We assumed that it takes at least 6 months for a drug to show a robust effect, so we used 6-month to separate the short and longterm effects.

For the secondary outcomes, we analyzed events of impaired liver function, gastrointestinal events, $\mathrm{CV}$ events, skin reaction, and musculoskeletal events. Details are shown in Appendix 2.

\section{Quality Assessment of Methods}

We used Cochrane Risk of Bias Tool to assess the quality and risk of bias for the included studies (Appendix 3). We defined the risk of bias as adequate, unclear, or inadequate for assessing 6 aspects of the trials: sequence generation, allocation concealment, blinding of participants and personnel, blinding of outcome assessment, incomplete outcome data, and selective reporting. The assessment was conducted by 2 independent reviewers, with a third consulted for resolution of any disagreements.

\section{Statistical Analysis}

We used "meta," "netmeta" and "dmetar" packages for the free statistical software $\mathrm{R}$ (version 4.0.3, Vienna, Austria) to undertake a frequentist pairwise meta-analysis and NMA.

NMA uses both direct and indirect evidence to compare multiple interventions within a statistical model. If 2 interventions have never been compared head-to-head, but both have been compared with a common comparator (such as placebo), an indirect comparison can be evaluated via the common comparator. ${ }^{25}$ An estimate of mean difference (MD) in treatment effect between 2 interventions is a weighted average of direct and indirect comparisons, with confidence intervals (CI).

For each primary outcome, we created network plot which shows the overall structure of comparisons in the NMA. The size of the circles is proportional to the number of patients randomized to each intervention, and the width of the edges is proportional to the number of studies making each comparison.

We had also performed pairwise meta-analyses of all head-to-head comparisons to evaluate the heterogeneity within each comparison. ${ }^{25}$

For continuous outcomes, such as serum uric acid level, eGFR, and BP, we estimated the difference in mean changes between the treatment and control groups. If a trial did not report such a result, we would calculate the difference in the follow-up measurements between 2 groups at a specific time point. We used the recommended methods by the Cochrane Handbook to impute missing values. ${ }^{26}$ League tables were created to summarize the results of pairwise comparisons from NMA. If a trial reported 2 or more results within the period, we used data of the shortest follow-up for short-term analysis and the longest follow-up for long-term analysis to distinguish the short-term and long-term effects better. For dichotomous outcomes, such as safety outcomes, we used the Peto odds ratio model because the event numbers were small or even zero in some studies. ${ }^{26}$ The study effect sizes were then synthesized using a random-effects NMA model.

To rank the treatments for each outcome, we used P-score, which measures how likely a treatment is better than the other competing treatments. $\mathrm{P}$-scores are derived from the $P$ values of pairwise comparisons for a treatment is compared with the other treatments in the network. P-scores reflect the differences between the point estimates of treatment effects but also take the precision into account. The range of $\mathrm{P}$-scores is from 0 to 1 , and a large P-score (eg, >0.90) suggests a high certainty of a treatment being more effective or safer than others. ${ }^{27}$ However, P-scores are descriptive, and a large difference between $2 \mathrm{P}$-scores does not necessarily mean the difference between the 2 treatments is statistically significant. There is no formal method to test the difference in $\mathrm{P}$-scores either.

If both direct and indirect evidence is available for a comparison between 2 treatments, we use the 
design-by-treatment interaction model and nodesplitting model to evaluate the consistency between direct and indirect evidence. We evaluated the assumption of transitivity for indirect comparisons by examining the distribution of confounding variables, such as baseline kidney function, or undertook subgroup analyses if the number of included studies is sufficient to conduct such analyses.

\section{Results}

Our literature search identified 777 potentially eligible studies. Thirteen randomized controlled trials were finally included in our systematic review, totaling 2842 people. $^{28-40}$ Figure 1 shows the study selection process in detail. Table 1 outlines the basic characteristics of the included studies. The intervention included allopurinol, benzbromarone, and febuxostat. The results of a pairwise meta-analysis on direct comparisons are shown in Appendix 6. Most comparisons show no substantial heterogeneity between studies.

\section{Primary Outcome}

\section{Short-Term Urate-Lowering Effect}

Eight studies were included in the analysis of the urate-lowering effect for short-term ( $\leq 6$ months) follow-up. ${ }^{29,30,32,33,35,37,39,40}$ The network plot and results of our NMA are summarized in Appendix 4 and Table 2. Patients used allopurinol, benzbromarone and febuxostat showed significantly lower serum uric acid level compared with placebo $(\mathrm{MD}=$ $-2.16 \mathrm{mg} / \mathrm{dL} ; 95 \% \mathrm{CI}, 3.2$ to -1.13 vs $\mathrm{MD}=$ $-3.05 \mathrm{mg} / \mathrm{dL} ; 95 \% \mathrm{CI},-5.19$ to -0.91 vs $\mathrm{MD}=$ $-2.71 \mathrm{mg} / \mathrm{dL} ; 95 \% \mathrm{CI},-3.9$ to -1.52 ), but there were no significant differences between drugs. Benzbromarone had the highest P-score of being ranked first for urate-lowering efficacy (Table 3).

\section{Long-Term Urate-Lowering Effect}

Three studies reported a long-term ( $>6$ months) urate-lowering effect. ${ }^{28,31,40}$ The network plot and results of our NMA are summarized in Appendix 4 and Table 2. Patients using allopurinol had significantly lower serum uric acid level compared with placebo $(\mathrm{MD}=-3.17 \mathrm{mg} / \mathrm{dL} ; 95 \% \mathrm{CI},-5.19$ to -1.15). Patients using febuxostat had lower blood uric acid levels (but not significantly different) compared with placebo. The serum uric acid level showed no significant difference between drugs.
Allopurinol had the highest P-score of being ranked first for better urate-lowering efficacy (Table 3).

\section{Renal Function: Short-Term Follow-up}

Five studies were included in this analysis. ${ }^{30,32,34,37,39}$ The intervention included allopurinol group and febuxostat group, and the network plot and results of our NMA are summarized in Appendix 4 and Table 2. Patients used allopurinol had significantly higher eGFR compared with placebo $(\mathrm{MD}=3.07 \mathrm{~mL} / \mathrm{min} /$ $1.73 \mathrm{~m}^{2} ; 95 \%$ CI, 0.18 to 5.95$)$. Patients who used febuxostat had a higher eGFR (but not significantly different) compared with placebo. Besides, allopurinol group also had higher eGFR compared with febuxostat group, but no statistical significance was found. Allopurinol had the highest P-score of being ranked first for better renal function (Table 3).

\section{Renal Function: Long-Term Follow-up}

Three studies were included in this analysis. ${ }^{31,36,40}$ The intervention included allopurinol group and febuxostat group. Appendix 4 and Table 2 showed the network plot and results of our NMA. Patients used allopurinol had significantly higher eGFR than using febuxostat or placebo $(\mathrm{MD}=3.70 \mathrm{~mL} /$ $\mathrm{min} / 1.73 \mathrm{~m}^{2}$; $95 \% \mathrm{CI}, 1.94$ to 5.46 vs $\mathrm{MD}=$ $4.10 \mathrm{~mL} / \mathrm{min} / 1.73 \mathrm{~m}^{2}$; $95 \%$ CI, 2.66 to 5.54 ). Patients used febuxostat had higher eGFR than using placebo but without statistical significance. Allopurinol had the highest $\mathrm{P}$-score (Table 3).

\section{Blood Pressure: Short-Term Follow-up}

Three eligible studies were included, and the network plot and results of our NMA for systolic/diastolic BP are summarized in Appendix 4 and Table $2 .^{30,34,35}$ No significant difference in systolic/diastolic BP between groups was found. P-score was summarized in Table 3.

\section{Blood Pressure: Long-Term Follow-up}

Four studies were included, and Appendix 4 and Table 2 showed the network plot and results of our NMA. ${ }^{28,31,36,40}$ No significant difference of systolic/diastolic BP was found between groups, except patients in febuxostat group had $1.47 \mathrm{mmHg}$ statistically lower diastolic BP than patients in placebo group $(\mathrm{MD}=-1.47 \mathrm{mmHg} ; 95 \% \mathrm{CI},-2.91$ to $-0.04)$. P-score was summarized in Table 3. 
Figure 1. Flowchart of the process to identify eligible studies with reasons for inclusion or exclusion.

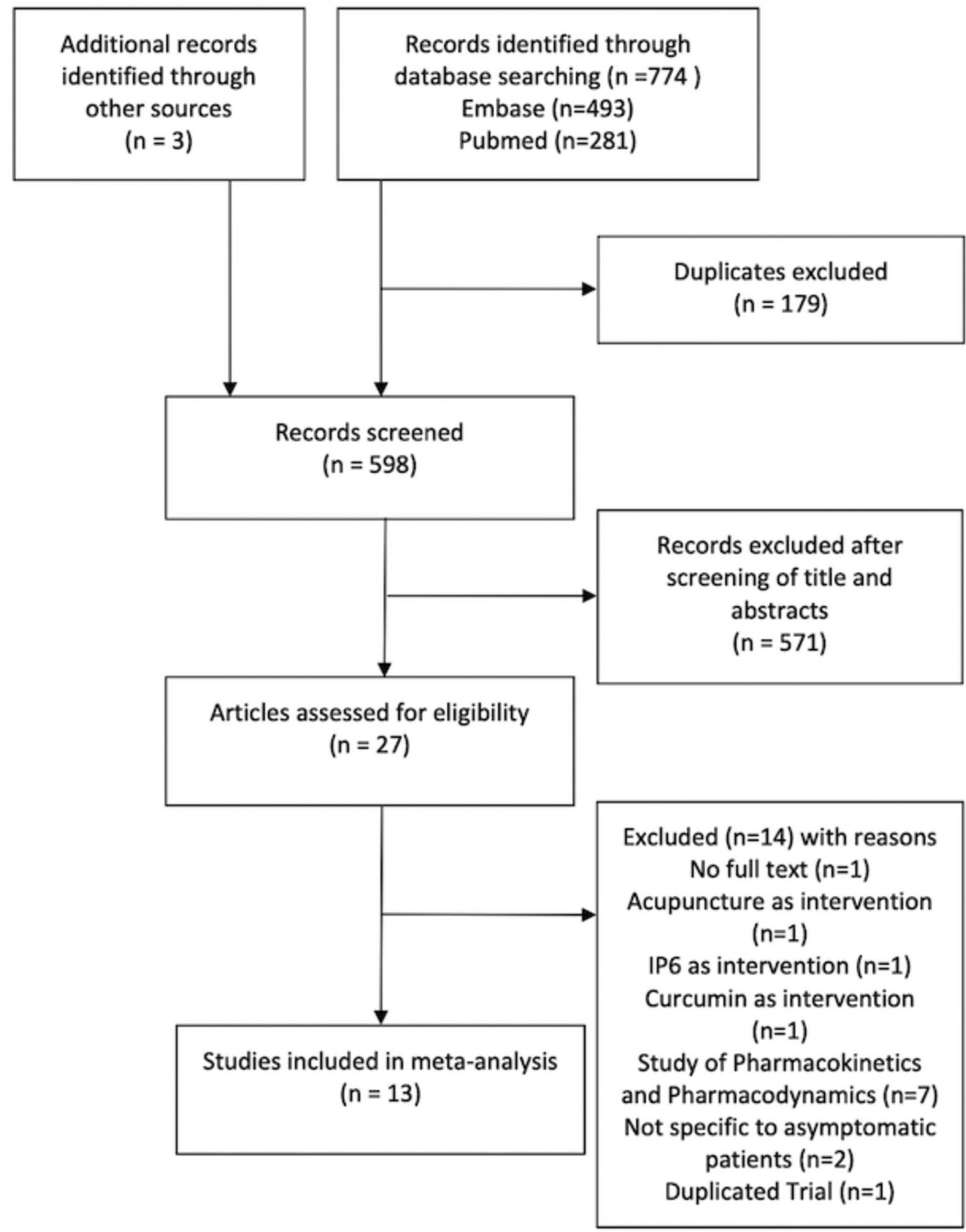




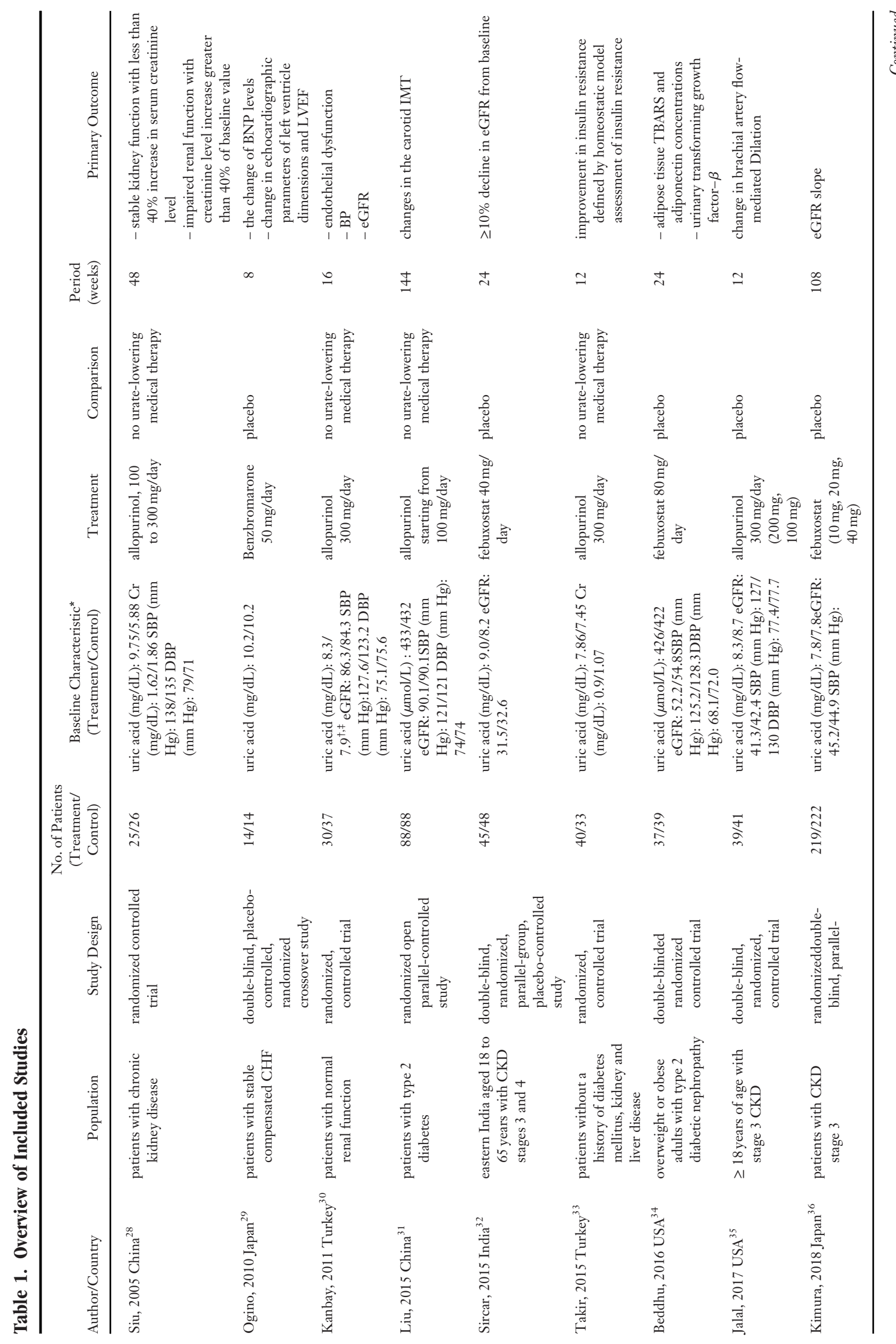




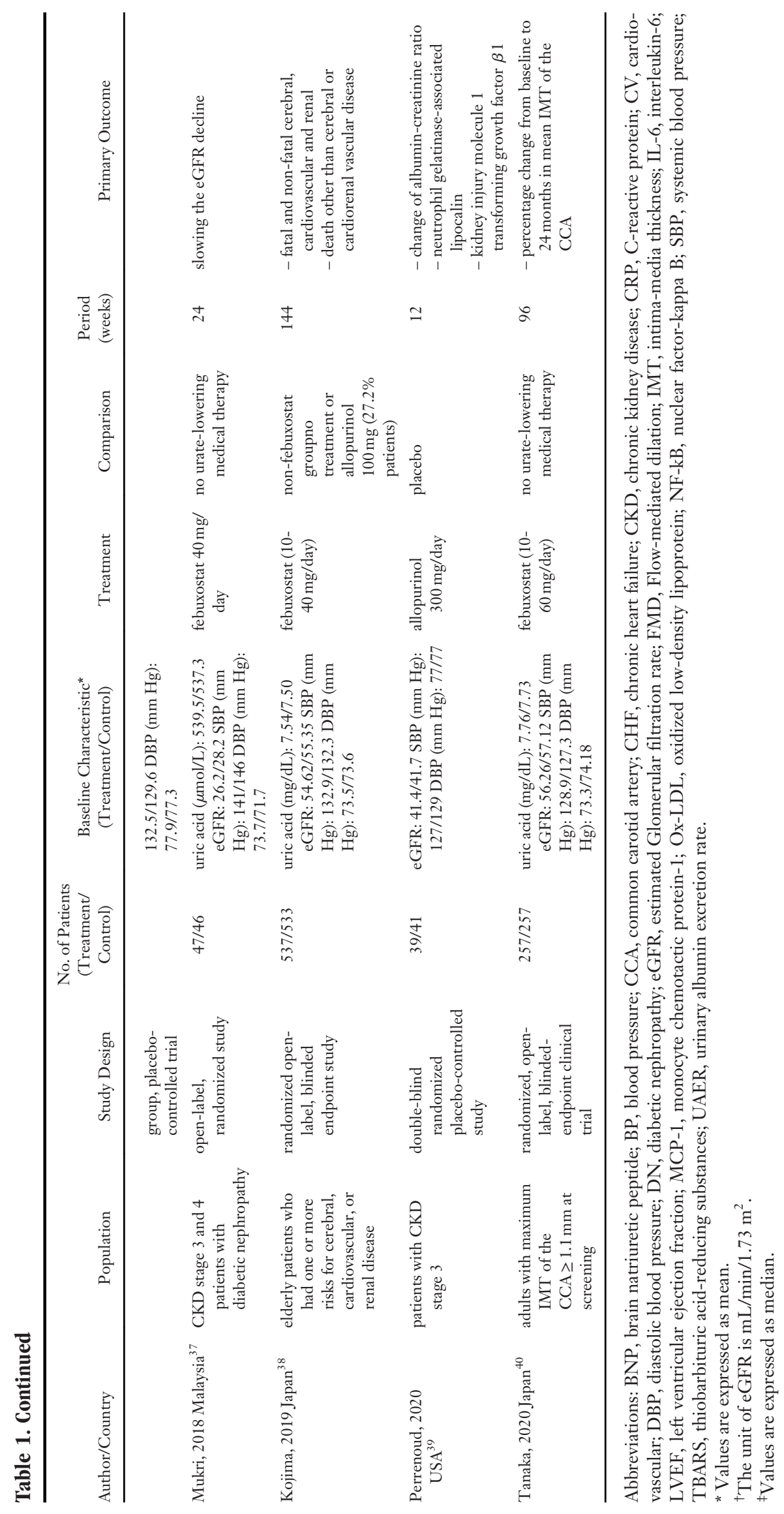


Table 2. League Table of Random-Effects Network Meta-Analysis for Effect of Urate-Lowering Therapy*

$\underline{\text { Serum Uric Acid Level (Short-Term Follow-Up, mg/dL) }}$

\begin{tabular}{llll}
\hline Allopurinol & . &. & $-2.16(-3.20$ to -1.13$)$ \\
$0.89(-1.49$ to 3.26$)$ & Benzbromarone &. & $-3.05(-5.19$ to -0.91$)$ \\
$0.55(-1.03$ to 2.13$)$ & $-0.34(-2.79$ to 2.11$)$ & Febuxostat & $-2.71(-3.90$ to -1.52$)$ \\
$-2.16^{\dagger}(-3.20$ to -1.13$)$ & $-3.05^{*}(-5.19$ to -0.91$)$ & $-2.71^{\dagger}(-3.90$ to -1.52$)$ & Placebo
\end{tabular}

Serum Uric Acid Level (Long-Term Follow-Up, mg/dL)

\begin{tabular}{lll}
\hline Allopurinol & $\cdot$ & $-3.17(-5.19$ to -1.15$)$ \\
$-0.55(-3.97$ to 2.88$)$ & Febuxostat & $-2.62(-5.39$ to 0.15$)$ \\
$-3.17^{\dagger}(-5.19$ to -1.15$)$ & $-2.62(-5.39$ to 0.15$)$ & Placebo
\end{tabular}

\begin{tabular}{lll}
\hline Renal Function (Short-Term Follow-Up, $\left.\mathrm{mL} / \mathrm{min} / 1.73 \mathrm{~m}^{2}\right)$ & $3.07(0.18$ to 5.95$)$ \\
\hline Allopurinol & \multicolumn{1}{c}{ Febuxostat } & $1.07(-2.43$ to 4.57$)$ \\
$2.00(-2.54$ to 6.53$)$ & $1.07(-2.43$ to 4.57$)$ & Placebo \\
$3.07^{\dagger}(0.18$ to 5.95$)$ &
\end{tabular}

$\underline{\text { Renal Function (Long-Term Follow-Up, } \mathrm{mL} / \mathrm{min} / 1.73 \mathrm{~m}^{2} \text { ) }}$

\begin{tabular}{lll}
\hline Allopurinol & $\cdot$ & $4.10(2.66$ to 5.54$)$ \\
$3.70^{\dagger}(1.94$ to 5.46$)$ & Febuxostat & $0.40(-0.60$ to 1.40$)$ \\
$4.10^{\dagger}(2.66$ to 5.54$)$ & $0.40(-0.60$ to 1.40$)$ & Placebo
\end{tabular}

$\underline{\text { Systolic Blood Pressure (Short-Term Follow-Up, mm Hg) }}$

\begin{tabular}{lll}
\hline Allopurinol & \multicolumn{1}{c}{} & $0.04(-4.22$ to 4.30$)$ \\
$4.54(-4.29$ to 13.37$)$ & Febuxostat & $-4.50(-12.23$ to 3.23$)$ \\
$0.04(-4.22$ to 4.30$)$ & $-4.50(-12.23$ to 3.23$)$ & Placebo
\end{tabular}

Systolic Blood Pressure (Long-Term Follow-Up, mm Hg)

\begin{tabular}{lll}
\hline Allopurinol & \multicolumn{1}{c}{} & $-4.74(-11.12$ to 1.63$)$ \\
$-3.96(-10.58$ to 2.66$)$ & Febuxostat & $-0.78(-2.57$ to 1.01$)$ \\
$-4.74(-11.12$ to 1.63$)$ & $-0.78(-2.57$ to 1.01$)$ & Placebo
\end{tabular}

$\underline{\text { Diastolic Blood Pressure (Short-Term Follow-Up, mm Hg) }}$

\begin{tabular}{llr}
\hline Allopurinol & $\cdot$ & $1.58(-2.31$ to 5.48$)$ \\
$1.48(-4.06$ to 7.03$)$ & Febuxostat & $0.10(-3.85$ to 4.05$)$ \\
$1.58(-2.31$ to 5.48$)$ & $0.10(-3.85$ to 4.05$)$ & Placebo \\
& & \\
\hline Diastolic Blood Pressure (Long-Term Follow-Up, mm Hg) & $\cdot$ & $-1.47(-2.90$ to -0.04$)$ \\
\hline Allopurinol & Febuxostat & Placebo \\
$2.34(-2.62$ to 7.29$)$ & $-1.47^{\dagger}(-2.91$ to -0.04$)$ &
\end{tabular}

*Data are shown as mean difference ( $95 \%$ confidence interval).

${ }^{\dagger}$ Difference in treatment effect is statistically significant. 
Table 3. P-Score of Different Rankings of Each Treatment Strategy

\begin{tabular}{lclc}
\hline \multicolumn{2}{c}{ SHORT-TERM } & \multicolumn{2}{c}{ LONG-TERM } \\
\hline \multicolumn{2}{c}{ Serum Uric Acid } & \multicolumn{2}{c}{ Serum Uric Acid } \\
Benzbromarone & 0.7908 & Allopurinol & 0.8108 \\
Febuxostat & 0.7146 & Febuxostat & 0.6728 \\
Allopurinol & 0.4937 & Placebo & 0.0164 \\
Placebo & 0.0009 & \multicolumn{2}{c}{ Renal Function } \\
\multicolumn{2}{c}{ Renal Function } & Allopurinol & $1.0000^{*}$ \\
Allopurinol & 0.8937 & Febuxostat & 0.3912 \\
Febuxostat & 0.4598 & Placebo & 0.1088 \\
Placebo & 0.1465 & Systolic Blood Pressure \\
\multicolumn{2}{c}{ Systolic Blood Pressure } & Allopurinol & $0.9035^{*}$ \\
Febuxostat & 0.8581 & Febuxostat & 0.4626 \\
Allopurinol & 0.3246 & Placebo & 0.1340 \\
Placebo & 0.3173 & Diastolic Blood Pressure \\
\multicolumn{2}{c}{ Diastolic Blood Pressure } & Febuxostat & 0.9000 \\
Placebo & 0.6534 & Placebo & 0.3307 \\
Febuxostat & 0.5900 & Allopurinol & 0.2693 \\
Allopurinol & 0.2567 & & \\
\hline
\end{tabular}

*Large value of $\mathrm{P}$-score $(\mathrm{eg},>0.90)$ may reflect that treatment is quite certain to be the most efficacious or safest.

\section{Secondary Outcome: Adverse Events}

Six trials, 1269 patients, were included in the analysis of impaired liver function..$^{29,31,34-36,40}$ Six trials, 986 patients, were included in the analysis of gastrointestinal events. ${ }^{31,32,34,35,37,40}$ Five trials, 1195 patients, were included in the analysis of cardiovascular event. ${ }^{32,34,36,37,40}$ Four trials, 1102 patients, were included in the analysis of musculoskeletal events. $^{34,36,37,40}$ Three trials, 1009 patients, were included in the analysis of skin reaction $35,36,40$ Compared with placebo via NMA, ULT did not significantly increase the odds of any secondary outcome (Appendix 5).

As no treatment groups formed a loop in any outcomes, we could not evaluate inconsistency between direct and indirect evidence. No subgroup analysis was undertaken because the number of the low number of included studies. Baseline eGFR of patients showed quite a wide variation across the included trials, but the assumption of transitivity was not considered seriously violated due to the hepatic metabolism of febuxostat, and both similar and typical dose was used in most trials of allopurinol. ${ }^{28,30,31,33,35,39}$

\section{Discussion}

Our NMA showed that benzbromarone and allopurinol have the best efficacy on lowering serum uric acid levels in short-term and long-term followup within AH patients. Patients using allopurinol have better eGFR than using placebo. ULT seems to have no significant effect on $\mathrm{BP}$, except for febuxostat on diastolic BP after long-term treatment. ULT does not significantly increase the risk of safety outcomes. Asymptomatic patients are often neglected for treatment, and our results provide much-needed evidence for treating those patients to attain better renal function.

\section{Uric Acid}

Previous meta-analysis or NMA included patients who were mostly symptomatic, so the doses of their drugs were relatively larger than those we recruited. Li et al reported a NMA for comparing efficacy of ULT in patients with or without gout. ${ }^{41}$ Their results showed benzbromarone (100 to $200 \mathrm{mg} /$ day) had better urate-lowering effect than allopurinol (100 to $600 \mathrm{mg} /$ day), and allopurinol (100 to $600 \mathrm{mg} /$ day) had better urate-lowering effect than febuxostat $(20 \mathrm{mg} /$ day $)$. In our NMA, only 1 trial reported the result of benzbromarone with a dose of $50 \mathrm{mg} /$ day, but we still found a similarly strong effect of benzbromarone in the short-term followup. However, no trial on benzbromarone reported results with more than 6 months of follow-up, so its long-term efficacy is uncertain. Our result showed that allopurinol (starting from $100 \mathrm{mg} /$ day) had better effect on lowering serum uric acid levels than febuxostat (10 to $60 \mathrm{mg} /$ day) in the long term. This result partly agrees with what $\mathrm{Li}$ et al found that allopurinol had a better effect than a low dose of febuxostat. $^{41}$

Nevertheless, the effect on uric acid is related to the dose of drugs. The selection of drugs and their doses also depends on patients' kidney function, responses to the treatment, and other factors.

\section{Renal Function}

Meta-analysis by Kanji et al showed patients with CKD using ULT had significantly better eGFR with a mean difference of $3.2 \mathrm{~mL} / \mathrm{min} / 1.73 \mathrm{~m}^{2}$ than using placebo. ${ }^{42}$ Slower eGFR decline rate by $4.1 \mathrm{~mL} / \mathrm{min} / 1.73 \mathrm{~m}^{2}$ per year compared with control group was found in the study of $\mathrm{Su}$ et $\mathrm{al}^{43}$ Those meta-analyses focused on patients with CKD and were not limited to asymptomatic patients. Our NMA included more diverse population, not only patients with $\mathrm{CKD}$, but the result still showed that patients using allopurinol had 3.07/ 
$4.1 \mathrm{~mL} / \mathrm{min} / 1.73 \mathrm{~m}^{2}$ significantly higher eGFR than using placebo in short-term/long-term follow-up. Although the differences are small, they may be of great significance for patients who already have kidney disease. In addition, the results were similar to previous research. ${ }^{42,43}$

Our result showed that febuxostat yielded a nonsignificant increase in eGFR compared with placebo. This was similar to a meta-analysis by $\mathrm{Li}$ et al which included symptomatic and asymptomatic CKD patients. ${ }^{44}$ As only 3 trials were included in their meta-analysis and 5 trials included in ours; these nonsignificant benefits may become significant if the number of subjects increases.

We did not find any trial of uricosuric agents reporting renal function of asymptomatic patients, so we cannot distinguish the possibly different effect between xanthin oxidase inhibitors and uricosuric agents.

\section{Blood Pressure}

The meta-analysis by Qu et al found allopurinol found a greater reduction in systolic BP and diastolic BP. ${ }^{45}$ They included patients with hyperuricemia with or without symptoms, so the dose of allopurinol $(100 \mathrm{mg} /$ day to $900 \mathrm{mg} /$ day $)$ was relatively larger than our studies. This may explain why allopurinol showed smaller effects on BP in our analysis. We found a decreasing trend of systolic BP under treatment of allopurinol and febuxostat in the long-term follow-up, but the effect of ULT on $\mathrm{BP}$ needs more research.

\section{Safety}

White et al found that in patients with gout and major CV coexisting conditions, using febuxostat showed higher all-cause mortality and CV mortality than using allopurinol in a median of 32 months in 6190 patients. ${ }^{17}$ Five trials, totaling 1195 patients, were recruited in our NMA reporting CV events. ${ }^{32,34,36,37,40}$ The result showed patients using febuxostat did not have a higher risk than those using placebo. However, no allopurinol-related trial was included in our analysis, so we could not compare the effects of these 2 drugs on CV events. The longest follow-up period in these trials was 27 months, but $\mathrm{CV}$ events may require more time and more patients to observe.

Allopurinol is frequently associated with StevensJohnson syndrome (SJS) or toxic epidermal necrolysis (TEN). ${ }^{15}$ Three trials in our NMA, totaling 1009 patients, reported skin reaction and did not show a higher risk of skin reaction in patients using allopurinal. ${ }^{35,36,40}$ Previous reports showed that the incidence rates of SJS/TEN range from 1.4 to 12.7 cases per million person-years. ${ }^{46,47}$ Therefore, such serious skin reaction is rare if the patient number is not large enough.

\section{Strengths and Limitations}

The strength of our NMA was that we focused on patients with $\mathrm{AH}$ and compared the efficacy of individual drugs. We also divided the treatment duration into short-term and long-term. However, this study has some limitations. First, only 3 drugs, allopurinol, febuxostat, and benzbromarone, were included in our analyses, while probenecid, lesinurad, and other urate-lowering drugs were not because these drugs had not been studied among AH patients. Second, no head-to-head trials that compared allopurinol and febuxostat were included in our analysis. Although it is the advantage of NMA that an indirect comparison can still be undertaken for these 2 treatments as both have been compared with placebo, we cannot verify the results because we do not have data from a direct comparison. ${ }^{48}$ Thirdly, the number of the included studies was too few to undertake subgroup analysis. For instance, only 1 trial focusing on CKD population was included in the analysis of long-term renal function, so we could not compare the efficacy of those drugs on renal function among CKD patients. In our NMA, the included trials recruited patients of different comorbidities. However, considering the kidney plays a major role in uric acid homeostasis, we felt that renal function was the most important factor, and we noted that the average eGFR of each trial in our analysis was different. Febuxostat undergoes hepatic metabolism, and its dose adjustment and effects are less affected by patients' renal function.49 Trials on allopurinol used similar doses, 200 to $300 \mathrm{mg} / \mathrm{day},{ }^{50}$ and this range of dose is considered suitable for CKD patients included in our NMA. ${ }^{51}$ Although the heterogeneous populations should be considered in the interpretation of our results, we felt that the assumption of transitivity was not seriously violated. Fourthly, our results showed Allopurinol has a renoprotective effect, and this finding seems quite robust in Asian population as our results were mainly derived from Asian studies. More randomized controlled trials from non-Asian countries are required to verify the protective effect. 


\section{Conclusions}

Our result showed that in AH patients, benzbromarone and allopurinol have the best urate-lowering effect in the short-term and long-term followup. Allopurinol has a significant renoprotective effect. Febuxostat has a significant effect on lowering diastolic BP in long-term follow-up. ULT does not result in a higher risk of impaired liver function, gastrointestinal event, $\mathrm{CV}$ event, skin reaction, and musculoskeletal event. According to the above results, patients with AH may be treated with ULT to benefit from renal protection, and the use of allopurinol should be considered a priority.

To see this article online, please go to: http://jabfm.org/content/ 35/1/140.full.

\section{References}

1. Jelani Q-U-A, Norcliffe-Kaufmann L, Kaufmann H, Katz SD. Vascular endothelial function and blood pressure regulation in afferent autonomic failure. Am J Hypertens 2015;28:166-72.

2. Rajendran P, Rengarajan T, Thangavel J, et al. The vascular endothelium and human diseases. Int J Biol Sci 2013;9:1057-69.

3. Zhou Y, Zhao M, Pu Z, Xu G, Li X. Relationship between oxidative stress and inflammation in hyperuricemia: analysis based on asymptomatic young patients with primary hyperuricemia. Medicine 2018;97:e13108.

4. Hadi HAR, Carr CS, Al Suwaidi J. Al Suwaidi J. Endothelial dysfunction: cardiovascular risk factors, therapy, and outcome. Vasc Health Risk Manag 2005;1:183-98.

5. Zhen H, Gui F. The role of hyperuricemia on vascular endothelium dysfunction. Biomed Rep 2017; 7:325-30.

6. Khosla UM, Zharikov S, Finch JL, et al. Hyperuricemia induces endothelial dysfunction. Kidney Int 2005;67:1739-42.

7. Terkeltaub R. Update on gout: new therapeutic strategies and options. Nat Rev Rheumatol 2010; 6:30-8.

8. Liang WY, Zhu XY, Zhang JW, Feng XR, Wang YC, Liu ML. Uric acid promotes chemokine and adhesion molecule production in vascular endothelium via nuclear factor-kappa B signaling. Nutr Metab Cardiovasc Dis 2015;25:187-94.

9. Xin W, Mi S, Lin Z. Allopurinol therapy improves vascular endothelial function in subjects at risk for cardiovascular diseases: a meta-analysis of randomized controlled trials. Cardiovasc Ther 2016;34:441-9.

10. Tsuruta Y, Kikuchi K, Tsuruta Y, et al. Febuxostat improves endothelial function in hemodialysis patients with hyperuricemia: a randomized controlled study. Hemodial Int 2015;19:514-20.
11. Cha R-H, Kim SH, Bae EH, et al. Physicians' perceptions of asymptomatic hyperuricemia in patients with chronic kidney disease: a questionnaire survey. Kidney Res Clin Pract 2019;38:373-81.

12. Mount DB. Asymptomatic hyperuricemia. Updated Jun 29, 2020. Accessed Jun 2, 2021.

13. Shin DH. To treat or not to treat asymptomatic hyperuricemia in chronic kidney disease. Kidney Res Clin Pract 2019;38:257-59.

14. Chalès G. How should we manage asymptomatic hyperuricemia? Joint Bone Spine 2019;86:437-43.

15. Halevy S, Ghislain PD, Mockenhaupt M, et al. Allopurinol is the most common cause of StevensJohnson syndrome and toxic epidermal necrolysis in Europe and Israel. J Am Acad Dermatol 2008; 58:25-32.

16. Becker MA, Schumacher HR, Jr., Wortmann RL, et al. Febuxostat compared with allopurinol in patients with hyperuricemia and gout. N Engl J Med 2005;353:2450-61.

17. White WB, Saag KG, Becker MA, CARES Investigators, et al. Cardiovascular safety of febuxostat or allopurinol in patients with gout. $\mathrm{N}$ Engl J Med 2018;378:1200-10.

18. Zhang W, Doherty M, Bardin T, EULAR Standing Committee for International Clinical Studies Including Therapeutics, et al. EULAR evidence based recommendations for gout. Part II: Management. Report of a task force of the EULAR Standing Committee for International Clinical Studies Including Therapeutics (ESCISIT). Ann Rheum Dis 2006;65:1312-24.

19. Love BL, Barrons R, Veverka A, Snider KM. Urate-lowering therapy for gout: focus on febuxostat. Pharmacotherapy 2010;30:594-608.

20. Azevedo VF, Kos IA, Vargas-Santos AB, da Rocha Castelar Pinheiro G, dos Santos Paiva E. Benzbromarone in the treatment of gout. Adv Rheumatol 2019;59:37.

21. Lee MH, Graham GG, Williams KM, Day RO. A benefit-risk assessment of benzbromarone in the treatment of gout. Was its withdrawal from the market in the best interest of patients? Drug Saf 2008;31:643-65.

22. Yamanaka H, Metabolism TG. Essence of the revised guideline for the management of hyperuricemia and gout. Japan Med Assoc J 2012; 55:324-9.

23. George J, Carr E, Davies J, Belch J, Struthers A. High-dose allopurinol improves endothelial function by profoundly reducing vascular oxidative stress and not by lowering uric acid. Circulation 2006;114:2508-16.

24. Kim S, Kim H-J, Ahn H-S, et al. Renoprotective effects of febuxostat compared with allopurinol in patients with hyperuricemia: a systematic review and meta-analysis. Kidney Res Clin Pract 2017;36:274-81. 
25. Rouse B, Chaimani A, Li T. Network meta-analysis: an introduction for clinicians. Intern Emerg Med 2017;12:103-11.

26. Higgins Jpt TJ, Chandler J, Cumpston M, Li T, Page MJ, Welch VA. Cochrane Handbook for Systematic Reviews of Interventions. Available at: https:// handbook.cochrane.org. Accessed May 11, 2021.

27. Maiuolo J, Oppedisano F, Gratteri S, Muscoli C, Mollace V. Regulation of uric acid metabolism and excretion. Int J Cardiol 2016;213:8-14.

28. Siu Y-P, Leung K-T, Tong MK-H, Kwan T-H. Use of allopurinol in slowing the progression of renal disease through its ability to lower serum uric acid levels. Am J Kidney Dis 2006;47:51-9.

29. Ogino K, Kato M, Furuse Y, et al. Uric acid-lowering treatment with benzbromarone in patients with heart failure: a double-blind placebo-controlled crossover preliminary study. Circ Heart Fail 2010;3:73-81.

30. Kanbay M, Huddam B, Azak A, et al. A randomized study of allopurinol on endothelial function and estimated glomular filtration rate in asymptomatic hyperuricemic subjects with normal renal function. Clin J Am Soc Nephrol 2011;6:1887-94.

31. Liu P, Wang H, Zhang F, Chen Y, Wang D, Wang Y. The effects of allopurinol on the carotid intima-media thickness in patients with type 2 diabetes and asymptomatic hyperuricemia: a three-year randomized parallelcontrolled study. Intern Med 2015;54:2129-37.

32. Sircar D, Chatterjee S, Waikhom R, et al. Efficacy of febuxostat for slowing the GFR decline in patients with CKD and asymptomatic hyperuricemia: a 6month, double-blind, randomized, placebo-controlled trial. Article. American Journal of Kidney Diseases 2015;66:945-50.

33. Takir M, Kostek O, Ozkok A, et al. Lowering uric acid with allopurinol improves insulin resistance and systemic inflammation in asymptomatic hyperuricemia. J Investig Med 2015;63:924-9.

34. Beddhu S, Filipowicz R, Wang B, et al. A randomized controlled trial of the effects of febuxostat therapy on adipokines and markers of kidney fibrosis in asymptomatic hyperuricemic patients with diabetic nephropathy. Can J Kidney Health Dis 2016;3:205435811667534.

35. Jalal DI, Decker E, Perrenoud L, et al. Vascular function and uric acid-lowering in stage $3 \mathrm{CKD}$. J Am Soc Nephrol 2017;28:943-52.

36. Kimura K, Hosoya T, Uchida S, FEATHER Study Investigators, et al. Febuxostat therapy for patients with stage $3 \mathrm{CKD}$ and asymptomatic hyperuricemia: a randomized trial. Am J Kidney Dis 2018;72:798-810.

37. Mukri MNA, Kong WY, Mustafar R, et al. Role of febuxostat in retarding progression of diabetic kidney disease with asymptomatic hyperuricemia: a 6months open-label, randomized controlled trial. Excli J 2018;17:563-75.
38. Kojima S, Matsui K, Hiramitsu S, et al. Febuxostat for Cerebral and CaRdiorenovascular Events PrEvEntion StuDy. Eur Heart J 2019;40:1778-86.

39. Perrenoud L, Kruse NT, Andrews E, et al. Uric acid lowering and biomarkers of kidney damage in CKD stage 3: a post hoc analysis of a randomized clinical trial. Kidney Med 2020;2:155-61.

40. Tanaka A, Taguchi I, Teragawa H, on behalf of the PRIZE study investigators, et al. Febuxostat does not delay progression of carotid atherosclerosis in patients with asymptomatic hyperuricemia: a randomized, controlled trial. PLoS Med 2020;17:e1003095.

41. Li S, Yang H, Guo Y, et al. Comparative efficacy and safety of urate-lowering therapy for the treatment of hyperuricemia: a systematic review and network meta-analysis. Sci Rep 2016;6:33082.

42. Kanji T, Gandhi M, Clase CM, Yang R. Urate lowering therapy to improve renal outcomes in patients with chronic kidney disease: systematic review and meta-analysis. BMC Nephrol 2015;16:58.

43. Su X, Xu B, Yan B, Qiao X, Wang L. Effects of uric acid-lowering therapy in patients with chronic kidney disease: a meta-analysis. PLOS ONE 2017;12: $\mathrm{e} 0187550$.

44. Lin T-C, Hung LY, Chen Y-C, et al. Effects of febuxostat on renal function in patients with chronic kidney disease: a systematic review and meta-analysis. Medicine 2019;98:e16311.

45. Qu LH, Jiang H, Chen JH. Effect of uric acid-lowering therapy on blood pressure: systematic review and meta-analysis. Ann Med 2017;49:142-56.

46. Hsu DY, Brieva J, Silverberg NB, Silverberg JI. Morbidity and mortality of Stevens-Johnson syndrome and toxic epidermal necrolysis in United States adults. J Invest Dermatol 2016;136:1387-97.

47. Diphoorn J, Cazzaniga S, Gamba C, REACTLombardia study group, et al. Incidence, causative factors and mortality rates of Stevens-Johnson syndrome (SJS) and toxic epidermal necrolysis (TEN) in northern Italy: data from the REACT registry. Pharmacoepidemiol Drug Saf 2016;25:196-203.

48. Jansen JP, Trikalinos T, Cappelleri JC, et al. Indirect treatment comparison/network meta-analysis study questionnaire to assess relevance and credibility to inform health care decision making: an ISPORAMCP-NPC Good Practice Task Force report. Value Health 2014;17:157-73.

49. Akimoto T, Morishita Y, Ito C, et al. Febuxostat for hyperuricemia in patients with advanced chronic kidney disease. Drug Target Insights 2014;8:39-43.

50. Harris MD, Siegel LB, Alloway JA. Gout and hyperuricemia. Am Fam Physician 1999;59:925-34.

51. Jodoin $\mathrm{K}$. The renal drug handbook: the ultimate prescribing guide for renal practitioners, 4th edition. Eur J Hosp Pharm 2016;23:248. 


\section{Appendices}

Appendix 1. Literature search strategy

Appendix 2. Data extraction from included trialsDetails of secondary outcomes

Appendix 3. Summary of the risks of bias in every included trial
Appendix 4. Network plot for effect of urate lowering therapy

Appendix 5. League table of the network meta-analysis comparing the events of secondary outcomes of all drugs

Appendix 6. Result of pairwise meta-analyses of all directly compared interventions 


\section{Embase}

\begin{tabular}{|c|c|c|}
\hline No. & Query & Results \\
\hline \#10 & \#8 AND \#9 & 493 \\
\hline \#9 & \#3 OR \#4 OR \#5 OR \#6 OR \#7 & 47551 \\
\hline \#8 & \#1 AND \#2 & 1574 \\
\hline \#7 & 'urate lowering therapy'/exp OR 'urate lowering therapy' OR (('urate'/exp OR urate) AND lowering AND ('therapy'/exp OR therapy)) & 1979 \\
\hline \#6 & $\begin{array}{l}\text { 'urate oxidase'/exp OR 'urate oxidase' OR (('urate'/exp OR urate) AND ('oxidase'/exp OR oxidase)) OR 'pegloticase'/exp OR pegloticase OR } \\
\text { 'rasburicase'/exp OR rasburicase }\end{array}$ & 5573 \\
\hline \#5 & $\begin{array}{l}\text { 'selective uric acid reabsorption inhibitor' OR (selective AND uric AND ('acid'/exp OR acid) AND reabsorption AND ('inhibitor'/exp OR inhibitor)) OR } \\
\text { 'lesinurad'/exp OR lesinurad }\end{array}$ & 364 \\
\hline \#4 & $\begin{array}{l}\text { 'uricosuric agent'/exp OR 'uricosuric agent' OR ('('uricosuric'/exp OR uricosuric) AND ('agent'/exp OR agent)) OR 'probenecid'/exp OR probenecid } \\
\text { OR 'benzbromarone'/exp OR benzbromarone OR 'sulfinpyrazone'/exp OR sulfinpyrazone }\end{array}$ & 18373 \\
\hline \#3 & 'xanthine oxidase inhibitor'/exp OR 'xanthine oxidase inhibitor' OR 'allopurinol'/exp OR allopurinol OR 'febuxostat'/exp OR febuxostat OR feburic & 27770 \\
\hline \#2 & asymptomatic & 245443 \\
\hline \#1 & 'hyperuricemia'/exp OR hyperuricemia OR 'uric acid'/exp OR 'uric acid' OR (uric AND ('acid'/exp OR acid)) & 7259 \\
\hline
\end{tabular}

\section{Pubmed}

\begin{tabular}{|c|c|c|c|c|c|}
\hline Search & Actions & Details & Query & Results & Time \\
\hline$\# 10$ & $\cdots$ & $>$ & $\begin{array}{l}\text { Search: (((hyperuricemia) OR (uric acid)) AND (asymptomatic)) } \\
\text { AND }((((((\text { xanthine oxidase inhibitor) OR (allopurinol)) OR } \\
\text { (febuxostat OR feburic)) OR (((uricosuric agent) OR } \\
\text { (probenecid)) OR (benzbromarone) OR (sulfinpyrazone))) OR } \\
\text { ((selective uric acid reabsorption inhibitor) OR (lesinurad))) OR } \\
\text { (((urate oxidase enzyme) OR (pegloticase)) OR (rasburicase))) } \\
\text { OR (urate lowering therapy)) }\end{array}$ & 281 & $20: 40: 22$ \\
\hline \#9 & $\cdots$ & $>$ & $\begin{array}{l}\text { Search: }((((((x a n t h i n e \text { oxidase inhibitor) OR (allopurinol)) OR } \\
\text { (febuxostat OR feburic)) OR ((((uricosuric agent) OR } \\
\text { (probenecid)) OR (benzbromarone)) OR (sulfinpyrazone))) OR } \\
\text { ((selective uric acid reabsorption inhibitor) OR (lesinurad))) OR } \\
\text { (((urate oxidase enzyme) OR (pegloticase)) OR (rasburicase))) } \\
\text { OR (urate lowering therapy) }\end{array}$ & 25,899 & $20: 40: 09$ \\
\hline \#8 & $\cdots$ & $>$ & Search: ((hyperuricemia) OR (uric acid)) AND (asymptomatic) & 819 & $20: 39: 45$ \\
\hline \#7 & $\cdots$ & $>$ & Search: urate lowering therapy & 3,314 & $20: 39: 30$ \\
\hline \#6 & $\cdots$ & $>$ & $\begin{array}{l}\text { Search: ((urate oxidase enzyme) OR (pegloticase)) OR } \\
\text { (rasburicase) }\end{array}$ & 2,347 & $20: 38: 37$ \\
\hline \#5 & $\cdots$ & $>$ & Search: (selective uric acid reabsorption inhibitor) OR (lesinurad) & 171 & $20: 37: 56$ \\
\hline \#4 & $\cdots$ & $>$ & $\begin{array}{l}\text { Search: (((uricosuric agent) OR (probenecid)) OR } \\
\text { (benzbromarone)) OR (sulfinpyrazone) }\end{array}$ & 8,810 & $20: 37: 17$ \\
\hline$\# 3$ & $\cdots$ & $>$ & $\begin{array}{l}\text { Search: ((xanthine oxidase inhibitor) OR (allopurinol)) OR } \\
\text { (febuxostat OR feburic) }\end{array}$ & 13,911 & $20: 36: 31$ \\
\hline \#2 & $\cdots$ & $>$ & Search: asymptomatic & 167,625 & $20: 35: 45$ \\
\hline \#1 & $\ldots$ & $>$ & Search: (hyperuricemia) OR (uric acid) & 43,504 & $20: 35: 13$ \\
\hline
\end{tabular}


Appendix 1. Literature search strategy

Impaired liver function

Liver dysfunction

Abnormal liver function test results

Gastrointestinal events

Vomiting

Diarrhea

Gastroenteritis

Loss of appetite

Melena

Nausea

Other gastrointestinal symptom or sign

CV events

Arrhythmia

Angina

Aortic aneurysm

Myocardial infarction

Heart failure

Other events related to CV system

Skin reactions

Skin eruption

Rash

Hypersensitivity

Dermatologic events

Musculoskeletal events

Joint pain

Fracture

Pain in back

Any musculoskeletal events 
Appendix 2. Data extraction from included trials: Details of secondary outcomes

\begin{tabular}{|c|c|c|c|c|c|c|}
\hline & 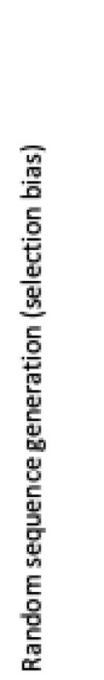 & 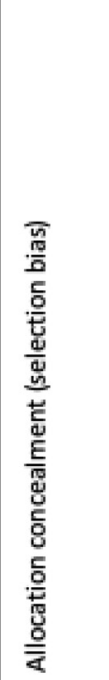 & 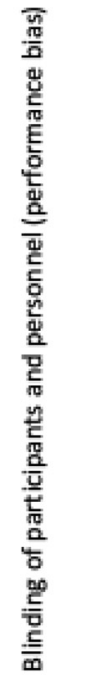 & 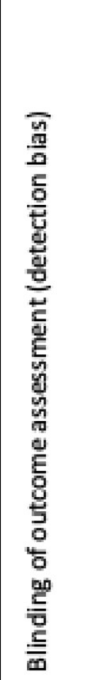 & 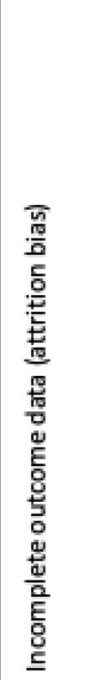 & 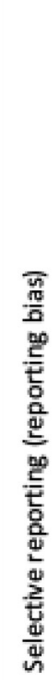 \\
\hline Siu, 2005 & Low & Unclear & Unclear & Unclear & Low & Low \\
\hline Ogino, 2010 & Low & Unclear & Low & Low & Low & Low \\
\hline Kanbay, 2011 & Low & Unclear & Unclear & Low & Low & Low \\
\hline Liu, 2015b & Low & High & High & Low & Low & Low \\
\hline Sircar, 2015 & Low & Unclear & Low & Low & Low & Low \\
\hline Takir, 2015 & Unclear & Unclear & Unclear & Unclear & Low & Low \\
\hline Beddhu, 2016 & Low & Low & Low & Low & unclear & Low \\
\hline Jalal, 2017 & Low & Low & Low & Low & Low & Low \\
\hline Kimura, 2018 & Low & Unclear & Low & Unclear & Low & Low \\
\hline Mukri, 2018 & Low & High & High & Low & Low & Low \\
\hline Kojima, 2019 & Low & High & High & Low & Low & Low \\
\hline Perrenoud, 2020 & Low & Unclear & Low & Unclear & Low & Low \\
\hline Tanaka, 2020 & Low & High & High & Low & Low & Low \\
\hline
\end{tabular}


Appendix 3. Summary of the risks of bias in every included trial

Serum uric acid analysis (short-term

follow-up)

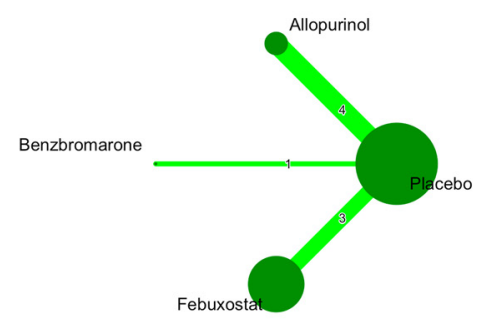

Renal function analysis (short-term

follow-up)

Allopurino
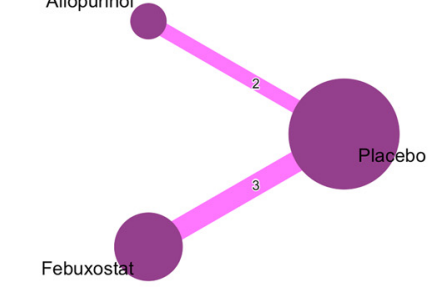

Systolic blood pressure (short-term follow-up)

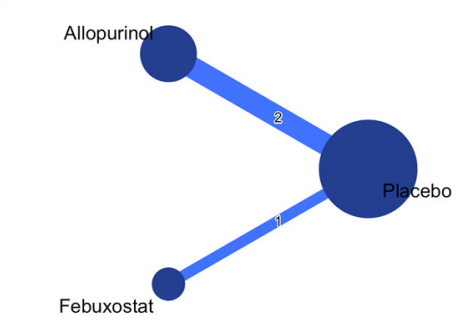

Diastolic blood pressure (short-term

follow-up)

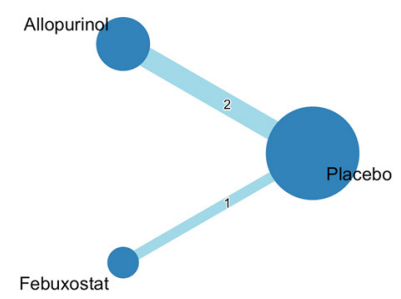

(Serum uric acid analysis (long-term

follow-up)

Allopurinol

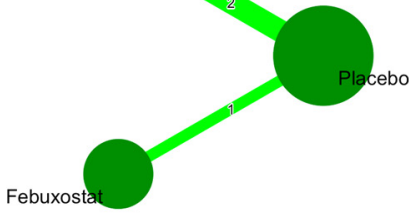

Renal function analysis (short-term follow-up)

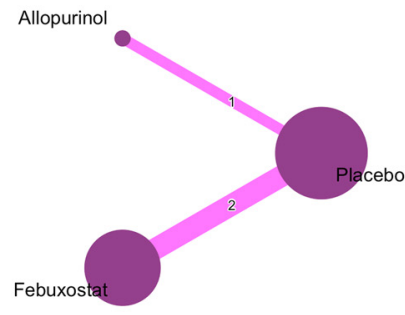

Systolic blood pressure (long-term follow-up)

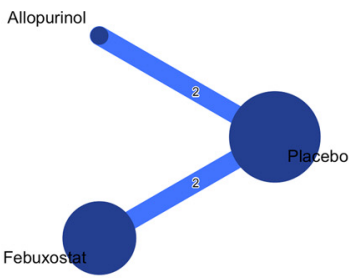

Diastolic blood pressure (long-term follow-up) 
Appendix 4. Network plot for effect of urate-lowering therapy. Each node represents a treatment group, and an edge indicates at least 1 trial comparing the 2 treatments on the ends of the edge. The node size in the network plot is proportional to the number of patients randomized to the treatment group, and the width of an edge is proportional to the number of studies making the pairwise comparison

\begin{tabular}{|c|c|c|}
\hline \multicolumn{3}{|c|}{ Impaired liver function } \\
\hline Allopurinol & - & $1.14(0.09 ; 15.25)$ \\
\hline $0.75(0.03 ; 18.30)$ & Febuxostat & $1.53(0.23 ; 9.92)$ \\
\hline $1.14(0.09 ; 15.25)$ & $1.53(0.23 ; 9.92)$ & Placebo \\
\hline \multicolumn{3}{|c|}{ Gastrointestinal events } \\
\hline Allopurinol & - & $2.65(0.53 ; 13.25)$ \\
\hline $0.87(0.10 ; 7.66)$ & Febuxostat & $3.05(0.70 ; 13.24)$ \\
\hline $2.65(0.53 ; 13.25)$ & $3.05(0.70 ; 13.24)$ & Placebo \\
\hline \multicolumn{2}{|c|}{ Cardiovascular events } & \\
\hline Febuxostat & $0.78(0.29 ; 2.07)$ & \\
\hline $0.78(0.29 ; 2.07)$ & Placebo & \\
\hline \multicolumn{3}{|c|}{ Skin reaction } \\
\hline Allopurinol & - & $0.14(0.00 ; 6.55)$ \\
\hline $0.06(0.00 ; 4.94)$ & Febuxostat & $2.24(0.28 ; 17.98)$ \\
\hline $0.14(0.00 ; 6.55)$ & $2.24(0.28 ; 17.98)$ & Placebo \\
\hline \multicolumn{2}{|c|}{ Musculoskeletal events } & \\
\hline Febuxostat & $2.08(0.69 ; 6.25)$ & \\
\hline $2.08(0.69 ; 6.25)$ & Placebo & \\
\hline
\end{tabular}

Appendix 5. League table of the network meta-analysis comparing the events of secondary outcomes of all drugs, including odds ratios and $95 \%$ confidence intervals

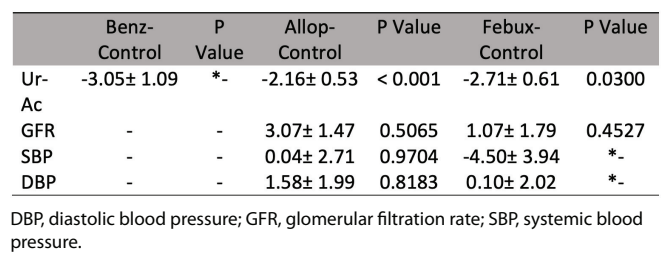


Result of pairwise meta-analyses of all directly compared interventions

Appendix 6.1 Results of pairwise meta-analyses of all directly compared interventions of short-term results. $P$ value is obtained from the Cochrane $Q$ test for heterogeneity.

\begin{tabular}{lcccc}
\hline & Allop-Control & $\boldsymbol{P}$ Value & Febux-Control & $\boldsymbol{P}$ Value \\
Ur-Ac & $-3.17 \pm 1.03$ & 0.0001 & $-2.62 \pm 1.41$ & $*_{-}$ \\
GFR & $4.10 \pm 0.73$ & $*_{-}$ & $0.40 \pm 0.51$ & 0.3547 \\
SBP & $-4.74 \pm 3.25$ & 0.6326 & $-0.78 \pm 0.91$ & 0.5233 \\
DBP & $0.86 \pm 2.42$ & 0.1484 & $-1.47 \pm 0.73$ & 0.8174 \\
\hline
\end{tabular}

*Only 1 trial included in the analysis so heterogeneity could not be evaluated.

thigh heterogeneity is defined by $\mathrm{P}$ value $<0.1$.

Appendix 6.2 Results of pairwise meta-analyses of all directly compared interventions of long-term results. $P$ value is obtained from the Cochrane $Q$ test for heterogeneity.

PRISMA NMA Checklist of Items to Include When Reporting A Systematic Review Involving a Network Meta-analysis

\begin{tabular}{|c|c|c|c|}
\hline Section/Topic & $\begin{array}{c}\text { Item } \\
\#\end{array}$ & Checklist Item & \begin{tabular}{|c} 
Reported on Page \\
$\#$ \\
\end{tabular} \\
\hline \multicolumn{4}{|l|}{ TITLE } \\
\hline Title & 1 & $\begin{array}{l}\text { Identify the report as a systematic review } \\
\text { incorporating a network meta-analysis (or related } \\
\text { form of meta-analysis). }\end{array}$ & 1 \\
\hline \multicolumn{4}{|l|}{ ABSTRACT } \\
\hline $\begin{array}{l}\text { Structured } \\
\text { summary }\end{array}$ & 2 & $\begin{array}{l}\text { Provide a structured summary including, as } \\
\text { applicable: } \\
\text { Background: main objectives } \\
\text { Methods: data sources; study eligibility criteria, } \\
\text { participants, and interventions; study appraisal; and } \\
\text { synthesis methods, such as network meta-analysis. } \\
\text { Results: number of studies and participants } \\
\text { identified; summary estimates with corresponding } \\
\text { confidence/credible intervals; treatment rankings } \\
\text { may also be discussed. Authors may choose to } \\
\text { summarize pairwise comparisons against a chosen } \\
\text { treatment included in their analyses for brevity. } \\
\text { Discussion/Conclusions: limitations; conclusions } \\
\text { and implications of findings. } \\
\text { Other: primary source of funding; systematic } \\
\text { review registration number with registry name. }\end{array}$ & $\begin{array}{l}\text { File of } \\
\text { JABFM_abstract }\end{array}$ \\
\hline \multicolumn{4}{|l|}{ INTRODUCTION } \\
\hline Rationale & 3 & $\begin{array}{l}\text { Describe the rationale for the review in the context of } \\
\text { what is already known, including mention of why a } \\
\text { network meta-analysis has been conducted. }\end{array}$ & 2 \\
\hline Objectives & 4 & $\begin{array}{l}\text { Provide an explicit statement of questions being } \\
\text { addressed, with reference to participants, } \\
\text { interventions, comparisons, outcomes, and study } \\
\text { design (PICOS). }\end{array}$ & 3 \\
\hline \multicolumn{4}{|l|}{ METHODS } \\
\hline $\begin{array}{l}\text { Protocol and } \\
\text { registration }\end{array}$ & 5 & $\begin{array}{l}\text { Indicate whether a review protocol exists and if and } \\
\text { where it can be accessed (e.g., Web address); and, if } \\
\text { available, provide registration information, including } \\
\text { registration number. }\end{array}$ & CRD42021256528 \\
\hline Eligibility criteria & 6 & $\begin{array}{l}\text { Specify study characteristics (e.g., PICOS, length of } \\
\text { follow-up) and report characteristics (e.g., years } \\
\text { considered, language, publication status) used as } \\
\text { criteria for eligibility, giving rationale. Clearly } \\
\text { describe eligible treatments included in the treatment } \\
\text { network, and note whether any have been clustered } \\
\text { or merged into the same node (with justification). }\end{array}$ & 3 \\
\hline
\end{tabular}


- Meta-regression analyses;

Alternative formulations of the treatment

network; and

- Use of alternative prior distributions for Userian analyses (if applicable).

RESULTS $\dagger$

\begin{tabular}{|c|c|c|c|}
\hline Study selection & 17 & $\begin{array}{l}\text { Give numbers of studies screened, assessed for } \\
\text { eligibility, and included in the review, with reasons } \\
\text { for exclusions at each stage, ideally with a flow } \\
\text { diagram. }\end{array}$ & 7 \\
\hline $\begin{array}{l}\text { Presentation of } \\
\text { network structure }\end{array}$ & S3 & $\begin{array}{l}\text { Provide a network graph of the included studies to } \\
\text { enable visualization of the geometry of the treatment } \\
\text { network. }\end{array}$ & Appendix 4 \\
\hline $\begin{array}{l}\text { Summary of } \\
\text { network geometry }\end{array}$ & S4 & $\begin{array}{l}\text { Provide a brief overview of characteristics of the } \\
\text { treatment network. This may include commentary on } \\
\text { the abundance of trials and randomized patients for } \\
\text { the different interventions and pairwise comparisons } \\
\text { in the network, gaps of evidence in the treatment } \\
\text { network, and potential biases reflected by the } \\
\text { network structure. }\end{array}$ & 6-10 \\
\hline $\begin{array}{l}\text { Study } \\
\text { characteristics }\end{array}$ & 18 & $\begin{array}{l}\text { For each study, present characteristics for which data } \\
\text { were extracted (e.g., study size, PICOS, follow-up } \\
\text { period) and provide the citations. }\end{array}$ & Table 1 \\
\hline $\begin{array}{l}\text { Risk of bias within } \\
\text { studies }\end{array}$ & 19 & $\begin{array}{l}\text { Present data on risk of bias of each study and, if } \\
\text { available, any outcome level assessment. }\end{array}$ & Appendix 3 \\
\hline $\begin{array}{l}\text { Results of } \\
\text { individual studies }\end{array}$ & 20 & $\begin{array}{l}\text { For all outcomes considered (benefits or harms), } \\
\text { present, for each study: 1) simple summary data for } \\
\text { each intervention group, and 2) effect estimates and } \\
\text { confidence intervals. Modified approaches may be } \\
\text { needed to deal with information from larger } \\
\text { networks. }\end{array}$ & $7-10$ \\
\hline Synthesis of results & 21 & $\begin{array}{l}\text { Present results of each meta-analysis done, including } \\
\text { confidence/credible intervals. In larger networks, } \\
\text { authors may focus on comparisons versus a } \\
\text { particular comparator (e.g. placebo or standard } \\
\text { care), with full findings presented in an appendix. } \\
\text { League tables and forest plots may be considered to } \\
\text { summarize pairwise comparisons. If additional } \\
\text { summary measures were explored (such as treatment } \\
\text { rankings), these should also be presented. }\end{array}$ & $\begin{array}{l}\text { Table 2,3 } \\
\text { Appendix } 5\end{array}$ \\
\hline $\begin{array}{l}\text { Exploration for } \\
\text { inconsistency }\end{array}$ & s5 & $\begin{array}{l}\text { Describe results from investigations of inconsistency. } \\
\text { This may include such information as measures of } \\
\text { model fit to compare consistency and inconsistency } \\
\text { models, } P \text { values from statistical tests, or summary of } \\
\text { inconsistency estimates from different parts of the } \\
\text { treatment network. }\end{array}$ & $\mathrm{NA} / 10$ \\
\hline $\begin{array}{l}\text { Risk of bias across } \\
\text { studies }\end{array}$ & 22 & $\begin{array}{l}\text { Present results of any assessment of risk of bias } \\
\text { across studies for the evidence base being studied. }\end{array}$ & Appendix 3 \\
\hline $\begin{array}{l}\text { Results of } \\
\text { additional analyses }\end{array}$ & 23 & $\begin{array}{l}\text { Give results of additional analyses, if done (e.g., } \\
\text { sensitivity or subgroup analyses, meta-regression } \\
\text { analyses, alternative network geometries studied, } \\
\text { alternative choice of prior distributions for Bayesian } \\
\text { analyses, and so forth). }\end{array}$ & $\mathrm{NA} / 10$ \\
\hline \multicolumn{4}{|l|}{ DISCUSSION } \\
\hline $\begin{array}{l}\text { Summary of } \\
\text { evidence }\end{array}$ & 24 & $\begin{array}{l}\text { Summarize the main findings, including the strength } \\
\text { of evidence for each main outcome; consider their } \\
\text { relevance to key groups (e.g., healthcare providers, } \\
\text { users, and policy-makers). }\end{array}$ & 11 \\
\hline Limitations & 25 & $\begin{array}{l}\text { Discuss limitations at study and outcome level (e.g., } \\
\text { risk of bias), and at review level (e.g., incomplete } \\
\text { retrieval of identified research, reporting bias). } \\
\text { Comment on the validity of the assumptions, such as } \\
\text { transitivity and consistency. Comment on any } \\
\text { concerns regarding network geometry (e.g., } \\
\text { avoidance of certain comparisons). }\end{array}$ & 14 \\
\hline Conclusions & 26 & $\begin{array}{l}\text { Provide a general interpretation of the results in the } \\
\text { context of other evidence, and implications for future } \\
\text { research. }\end{array}$ & 15 \\
\hline \multicolumn{4}{|c|}{$\mathrm{P}$} \\
\hline & 27 & $\begin{array}{l}\text { Describe sources of funding for the systematic review } \\
\text { and other support (e.g., supply of data); role of } \\
\text { funders for the systematic review. This should also } \\
\text { include information regarding whether funding has } \\
\text { been received from manufacturers of treatments in } \\
\text { the network and/or whether some of the authors are } \\
\text { content experts with professional conflicts of interest } \\
\text { that could affect use of treatments in the network. }\end{array}$ & $\begin{array}{l}\text { File of } \\
\text { JABFM_title page }\end{array}$ \\
\hline
\end{tabular}

PICOS = population, intervertion, comprato, oucones, sudy design * Text in italics indicateS wording specific to reporting of network meta-analyses that has been added to ta

of appendices to present all relevant information in full detail for items in this section. 